\title{
Management of Patients with Hemangiopericytoma: Case Report and Literature Review
}

\author{
Bruno Barreira Cardoso ${ }^{1}$, Daniely Pessoa Moreira ${ }^{1}$, Francisco de Assis Fernandes Tavares ${ }^{1}$, \\ Luiz Frederico Bezerra Honorato Junior ${ }^{1}$, Nicole Cindy Fonseca Santos ${ }^{1}$, \\ Rebecca Renata Lapenda do Monte ${ }^{1}$, Renan Cavalcante Souza ${ }^{1}$, Renata Carneiro Melo ${ }^{1}$, \\ Luciana de Aguiar Correa ${ }^{2}$, Renato Serquiz Elias Pinheiro ${ }^{3}$ \\ ${ }^{1}$ Universidade Potiguar, Natal, Brazil \\ ${ }^{2}$ Pediatrics Department, Hospital Infantil Varela Santiago, Natal, Brazil \\ ${ }^{3}$ Neurology Department, Universidade Potiguar, Natal, Brazil
}

\section{Email address:}

brunobarreicac@gmail.com(B.B. Cardoso),danielypessoamoreira@hotmail.com(D.P. Moreira), 95.tavares@gmail.com(F.de A. F. Tavares),fredhonoratojr@gmail.com(L.F. B. H. Junior), ncindyfs@gmail.com(N.C. F. Santos), rebeccalapenda3@gmail.com(R.R. L.do Monte),souza.renancavalcante@gmail.com(R.C. Souza), renatacarneiromelo@gmail.com(R.C.Melo), luaguiarcorrea@ig.com.br(L.de A. Correa), renatosep@hotmail.com(R. S. E. Pinheiro)

\section{To cite this article:}

Bruno Barreira Cardoso, Daniely Pessoa Moreira, Francisco de Assis Fernandes Tavares, Luiz Frederico Bezerra Honorato Junior, Nicole Cindy Fonseca Santos, Rebecca Renata Lapenda do Monte, Renan Cavalcante Souza, Renata Carneiro Melo, Luciana de Aguiar Correa, Renato Serquiz Elias Pinheiro. Management of Patients with Hemangiopericytoma: Case Report and Literature Review. American Journal of Health Research. Vol. 7, No. 2, 2019, pp. 24-30. doi: 10.11648/j.ajhr.20190702.12

Received: May 9, 2019; Accepted: July 3, 2019; Published: July 24, 2019

\begin{abstract}
The hemangiopericytomas (HPCs) are rare tumors of the dural base and correspond of less than $1 \%$ of all primary Central Nervous System (CNS) tumors. It usually affects adult males with 40 to 50 years old and has a supratentorial location $(70 \%)$, followed by posterior fossa $(15 \%)$ and spine $(15 \%)$. Generally, it has fast progression and manifests clinically through headache, focal neurological deficits and epileptic seizures. The tumor has high incidence of local recurrence, from $34 \%$ to $90 \%$, and it is estimated that the follow-up of these patients should be done for at least 7, 5 years. The percentage of longdistance metastases is lower, $12 \%$ to $55 \%$, affecting especially bones, lungs and liver, in decreasing order. The objective of this study is to evaluate the contradictions when approaching these tumors and to analyze a rare case of pediatric involvement. It was prepared a case report and a literature review was made using the data bases MEDLINE®, PubMed and Cochrane, where 31 relevant articles were selected related to the proposed study. The outcome of HPC treatment can change according to the tumor's location in the CNS or extracranial, the tumor's size and the histopathological classification. Therefore, many studies have been proposed observing the answer of the surgical resection, the adjuvant radiotherapy, the radiosurgery and the antiangiogenic therapies.
\end{abstract}

Keywords: Hemangioperycitoma, Radiosurgery, Radiotherapy, Surgery

\section{Introduction}

The hemangiopericytoma (HPC) is considered a variant from the extra pleural solitary fibrous tumor, a rare tumor of dural base, which has a large histological spectrum. It is responsible for less than $1 \%$ of all primary tumors of the CNS (Central Nervous System), and only 2, 1\% are HPC grade II, mainly occurring in adults between 40 and 50 years old with a small predominance on males $[1,2]$.
The distribution inside the CNS is similar to the meningioma, in which $70 \%$ are supratentorial, $15 \%$ are in the posterior fossa and $15 \%$ are in the spine. Intraventricular lesions were also reported. The extracranial metastatic disease more commonly occurs in organs like bones, lungs and liver [1]. Reports suggests that the average time for local recurrence is about 5 years, while the average for long 
distance metastases is about 8 years. This dissemination usually occurs both for lymphatic and hematogenic ways [2].

Inside the CNS, the hemangiopericytoma is typically intracranial and, as said before, it is originated from the dura mater. These tumors are clinically alike with meningioma; however, they exhibit a fast-growing time. They could cause symptoms due to its mass effect or local edema, with compression of the adjacent cerebral parenchyma and raising of intracranial pressure. In this way, they usually present symptoms such as headache, focal neurological deficits or epileptic seizures [3].

It has similarity with meningioma on computed tomography and magnetic resonance imaging, however its clinical behavior is more aggressive, and because of this your recurrence rate is up to $90 \%$ in 12 years [4].

Since its original description it has been a lot of variability on diagnostic criteria and histopathologic classification, but according to the actual graduation of 2016 World Health Organization (WHO), the HPC are grouped with the solitary fibrous tumor, in a way that grade I most often corresponds to the highly collagenous, relatively low cellularity, spindle cell lesion previously diagnosed as solitary fibrous tumor; grade II corresponds typically to the more cellular, less collagenous tumor with plump cells and "staghorn" vasculature that was previously diagnosed in the CNS as hemangiopericytoma; and grade III most often corresponds to what was termed anaplastic hemangiopericytoma in the past, diagnosed on the basis of 5 or more mitoses per 10 high-power fields [5].

The diagnosis may be suspected by clinical manifestations, as well as by neuroimaging exams, but the confirmation requires histopathological evaluation. It will be reported a case of congenital intracranial HPC in full term newborn whose tumor was successfully resected right after birth.

This literature review and case relate in a pediatric patient - with rare epidemiological feature - has the objective of discussing the main therapeutically ways for hemangiopericytoma management.

\section{Methods}

The data research platforms used for the selection of the articles analyzed in this review were: PubMed, MEDLINE $®$ and Cochrane Database. Were selected the articles related with management of hemangiopericytoma in the last 5 years, and the following search terms were used: "Intracranial Hemangiopericytoma", "Management" and "Treatment".

The studies considered relevant published between January 2014 and 2019 were included, being part of the following study genre: prospective and retrospective cohort, systematic and non-systematic review articles, study of case series and clinical trials. The filters used were: human studies, complete articles and without age restriction, totaling 41 articles. After analysis for choosing the articles corresponding to the objective of this study, 10 unsuitable ones were excluded, remaining 31 .

\section{Case Report}

Female patient, with 4 months and 13 days of life, born and coming from São Paulo do Potengi - RN. During prenatal care, with 37 weeks and 1 day pregnancy, was pointed by an obstetric ultrasonography having a bilateral lateral brain ventriculomegaly, measuring $1,54 \mathrm{~cm}$. However, the pregnancy proceeded with no intercurrences and the child-birth was full term. After the birth, the infant was admitted on the health service for detailed investigation of her Central Nervous System, and a Computerized Tomography had been done for enlighten of ultrasonographic alterations. Trough CT it could be seen a presence of a rounded mass, well defined, spontaneously denser than the brain parenchyma, located in the posterior fossa, close to the cerebral aqueduct (IV ventricle), causing supratentorial hydrocephaly associated with ependymal cerebrospinal fluid transudation. This injury is topographic found on the midline, causing deviation and compression of the cerebellar hemispheres, notably to the left, showing a hypodense area in its interior, and may be related to cystic degeneration (figure 1). Then, a total resection of the tumor was proceeded, and an anatomopathological and immunohistochemistry studies reveled a lesion compatible with hemangiopericytoma. The tumor's surgical histopathology review confirmed high mitotic rate of 7 mitoses per 10 high power fields and a proliferation index about $10 \%$. Upon biopsy analysis, tumor grade was grade III anaplastic HPC. After the surgery, the patient kept hospitalized in an intense care unity, evolving with infection and surgical wound dehiscence, positive blood culture for Klebsiella pneumoniae and a urinary tract infection by Staphylococcus aureus, treated with large specter antibiotic therapy. She developed severe dysphagia, bronchospasm, nasal hypersecretion and respiratory discomfort, and the diagnosis of gastroesophageal reflux disease was made, wherefore a gastrostomy and fundoplication were performed. The evolution of the condition followed up to be slow and with frequent intercurrences resulting from bronchial aspiration, besides weight loss. She was discharged from the hospital after 54 days of hospitalization. After 1 year of life the patient realized a Magnetic Resonance evidencing post-surgical alterations on posterior fossae, large supratentorial ventricular dilatation and inferior portion of cerebral aqueduct with reduced caliber. At this moment she has outpatient follow-up with good general status and adequate neuropsychomotor development for her age. 



Figure 1. Axial section of a cranial CT showing a mass of the Hemangiopericytoma.

\section{Discussion}

The word hemangiopericytoma (HPC) was described by Stout and Murray in 1942 for relating a rare neoplasm, originated from the Zimmerman pericytes, located in the skeletal muscle tissue and cutaneous tissue. It represents only $1.6 \%$ of all SNC tumors. Anatomically, the HPCs can happen in any location with capillaries $[2,6,7]$.

As said before, it has higher incidence in adults around the fortieth life decade, being uncommon in children. It affects 0.34 infants on 1 million born alive. According to the age, the HPCs are graduated in two clinical types: HPC in infants $(<1$ year old) and HPC in adults (> 1 year old) $[8,9]$.

Differently from what happens frequently with the adult forms of HPC, the most infants HPC are benign. Thus, with diagnosis and early approach, the clinical behavior is better in children, presenting good response to chemotherapy, surgery and even tendency of spontaneous regression [10].

For the newborns, it is recommended that, if there is good clinical conditions and high diagnosis suspicions, the surgery must be made as soon as possible. It is advocated the surgical removal of the tumor as first line treatment, but there is no concrete evidence of radiotherapy efficiency on intracranial HPC in infants, especially on infants diagnosed with less than 1 year old [8].

Despite the treatment of intracranial tumors on infants with less than 1 year old being currently approached, the prognosis still reserved [8].

The IHPC is an aggressive tumor with tendency for early loco regional recurrence and metastasis for different organs, including bone (19.6\%), lungs and pleura (18.4\%), liver $(17.6 \%)$ and spine $(14.1 \%)$. The intracranial tumor is rare and representes only $0.4 \%$ to $1 \%$ of all primary brain tumors, with only $10 \%$ being detected on the pediatric ages $[8,11,12]$.

Commonly, its symptoms are caused by the mass effect, but it is presented also with focal alterations, headache or convulsions. It rarely presents intracranial bleeding, since only 12 cases were described on the literature [9].

Microscopically, these lesions exhibit intra tumoral bleedind, because during the tumor growing there is erosion of blood vessels, distortion, distention, occlusion and necrosis due to endothelial proliferation. In these particular cases it is necessary the emergency surgery, as occurs in subarachnoid hemorrhage [9].

It is elementary to observe the tumor particularities for deciding the therapeutic choices and, in cases of aggressive tumor type, the treatment option should be less invasive and more palliative. Variables as age, tumor's size, number of mitoses (more than 5 per 10 field of high power), retroperitoneal localization and incomplete excision of the primary lesion are associated with a bad prognosis $[13,14]$.

Currently it could be part of the treatment for HPC: preoperative embolization, total or subtotal surgery resection of the tumor, adjuvant radiotherapy and radiosurgery. However, the chemotherapy do not show efficient in the treatment of HPCI, given its high aggressiveness, but can be considered in cases of peripheral metastasis [15].

To facilitate the surgery resection, a therapeutic choice is the preoperative embolization, improving the visualization and decreasing the blood loss. The indications for this procedure include: story of excessive bleeding in previous surgery; hypervascularity tumors; deep tumors with hard access on the early surgical access of the main feed vessels; tumors associated with intratumoral aneurysm. However, in HPCs bigger than $6 \mathrm{~cm}$ the embolization did not show intraoperative benefits [14-16].

Other preoperative option is the use of biomarkers to distinguish the pathological tissue from the non-tumoral tissue. The resection guided by 5 -aminoluvolenic acid (5ALA) from high grade gliomas showed superiority over regular white light, because the intraoperative identification of tumor regions by fluorescence guides the surgeon to resect the tumor's tissue that would not be visualized. Meanwhile, the 5-ALA seems to play a limited role in the resection of children's tumor and it is not recommended routinely, thus has no indication for the presented case [13].

The lesion's surgery resection is the pattern treatment for all HPC types. The global survival and the survival free of disease are usually more prolonged when the patients are submmited to Grossly Total Resection (GTR), in comparison with Partial Resection (PR). For this reason, the GTR is usually more widespread today [15].

Chen et al. made a study on patients with IHCP that were treated with GTR or PR. Beyond the surgical approach, in both cases an adjuvant radiotherapy (RT) was made that included Conventional Radiotherapy (CRT) and Stereotactic Radiosurgery (SRS), but no adjuvant chemotherapy approach was done. In this study, the GTR associated with a conventional adjuvant radiotherapy were the best options for the treatment [17]. 
It is important to emphasize that the analyzed studies showed that the chemotherapy as neoadjuvant or adjuvant treatment for IHPC was not recommended, because of the high resistance of the tumor to this treatment, but there may be indication in cases of peripheral metastatic disease [15].

In the other hand, Wang et al. made a cohort with 191 HPC and anaplastic HPC patients and concluded that IHPC cases should be approached with surgical resection of the tumor and postoperative radiotherapy, being the best way of retarding recurrence cases. Beyond that, the GTR should be done only in tumor bigger than $3 \mathrm{~cm}$. Thus, in patients that did not do the surgery, do have contraindications or a tumor smaller than $3 \mathrm{~cm}$, than the radiotherapy should be the best option [13].

Despite the common use of GTR as therapeutical option, the HPC presents a high local recurrence rate. Among those that relapse, the ones with diameter bigger than $6-7 \mathrm{~cm}$ reoccurs faster than the little ones. This happen because the bigger tumors are harder to be completely resected. Moreover, the tumors grade III also presented higher chances of reoccur, because shows fast cellular proliferation $[17,18,32]$.

Because of the high recurrence rate and the tumor gravity, aggressive and careful therapies should be proceeded, and a long term follow up should be done, for at least 2, 5 years, in order to avoid extracranial metastases. Most cases of extracranial metastases are underestimated in number, because the adequate screening is not commonly done on asymptomatic patients after surgical approach [19, 20, 21].

In a retrospective analysis, Jeon et al. demonstrated better local control of the tumor with the GTR followed by radiotherapy (RT), but there is no evidence on survival rate alteration. Thus, the postoperative radiotherapy (PORT) contributes for volumetric regression and parenchymal edema reduction, following up with improvement of the neurological symptoms on most patients, including the ones approached with PR. Although, it is advisable that the adjuvant RT should be carefully selected accordingly with the extension of the resection and pathological classification of the tumor [17, 18, 20, 22].

Ghia et al. made a retrospective study analyzing 63 patients and observed that there is better local control of the disease with the use of adjuvant RT on doses $\geq 60 \mathrm{~Gy}$. It was seen that patients that received doses $<50$ Gy presented marginal intracranial recurrences, concluding that the use of PORT gives great benefit, but do not exclude the risk of marginal or distant recurrence if used in low doses [3].

Choi $\mathrm{J}$ et al. also confirmed a superiority in the association of GTR and PORT, proving better results in front of other studies of the scientific community, but it observed that there is better survival rate, disagreeing with other previous studies. Also, they saw that certain histological markers, like Ki67 and the PHH3 expression, indicates a higher proliferation and tumoral grade. Thus, when these markers are present, it indicates a worse survival rate and tumor recurrences, and a better approach should be taking in consideration. Beyond these, the expression of PD-L1 also lower the survival rate $[23,24]$.
The use of PORT on raising the local control rate and global survival rate in patients treated with surgery is not related with the extension of the tumoral resection. This way, the association of PORT after GTR presents a tendency of improving the survival, free of regional metastases, but shows no effect preventing long distant metastases. The general recurrence rate was superior in patients that did not receive PORT, since the treatment with RT acts on local control of the disease, delaying the appearance of regional and distant metastases [20, 23].

Differently from others studies, which point the GTR as a better prognostic factor, the work from Lee et al did not get results correlating GTR with higher survival rates [20].

It was also evidenced that the tumor site is not the main factor to be observed, but it is necessary to locate the tumor precisely, because if there is a dural sinus or bone invasions, the realization of GTR becomes risky due to the hard anatomical accessibility, besides the high amount of intraoperative blood loss [17, 20].

It is common that HPC patients presents hydrocephaly with an obstructive disposition or a postoperative disposition, being necessary the use of neurosurgical procedures as ventriculoperitoneal shunt (VPS) or endoscopic third ventriculostomy (ETV) for the treatment of this complication. However, studies indicate that the usage of VPS is associated with a higher chance of extra neural metastases when compared with ETV. It is estimated that the time since the installation of the VPS to the identification of the distant metastases vary from 1 month to 3 years [15].

Thus, it is proposed that the patients with HPC associated with obstructive hydrocephaly that need decompression of the cerebrospinal fluid should be treated with the ETV in order to minimize the risk of metastases [15].

It is important to note that the surgery performed by Gamma Knife ${ }^{\circledR}$ (non-invasive stereotactic radiosurgery) has been shown to be a therapy with an unsatisfactory recurrence percentage and even a return of IHPC in a faster time [23].

On the other hand, Spina et al. analyzed this treatment for HPC through a case series, and concluded that it is a viable therapeutic option and effective for new, residual and recurrent tumors, since the doses are bigger than 14 Gy. Nevertheless, new studies should be focused on define the exactly paper of radiosurgery on the management of HPCI. The GTR is the treatment of choice against the PR, and after data analysis, it was decided that total surgical resection with radiotherapy is the best treatment with better progression-free survival rate and better action on disease recurrence $[25,26]$.

Kim et al., after review of 18 patients with HPC agrees that the GKS should be used for control of residual or recurrent tumors with good local control in short term. Even with high recurrence, the tumor's local control rate is high $(80 \%)$ and the extra GKS for recurrent lesions keeps as efficient as first GKS. Therefore, the repetition of this therapy in recurrent tumors can catch up the tumor's control in long-term [26].

Because of the high vascularization and the localization of the HPC, this lesion become easily detectable in magnetic 
resonance image. This exam can early identify relapses, intracranial metastases and long-distance metastases when it has the correct follow-up. Therefore, for postoperative control and to confirm the absence of tumor mass, magnetic resonance imaging is recommended, being an important factor evaluated in the follow-up of the patients [20,3].

Even there is no agreement about the ideal time of followup in patients with these rare tumors, it is recommended the long-term treatment, because of the unpredictable and aggressive behavior of the disease. Thus, it is important to strictly observe the patients' clinical signs, especially when it has complaints related to the main long-distance metastases sites, as bone pain [15].

Therefore, when comparing the treatments of isolated surgery, isolated radiotherapy and combination of surgery and radiotherapy, the treatment modality showed no significant impact on the disease specific survival in 5, 10 and 20 years of head and neck HPC, but has an impact on other hemangiopericytoma [27].

It is important to say that isolated surgical procedures are primary modalities, so that radiotherapy must have more studies in order to truly show its role. It should be considered in cases of positive or uncertain surgical margins [27].

\section{Conclusion}

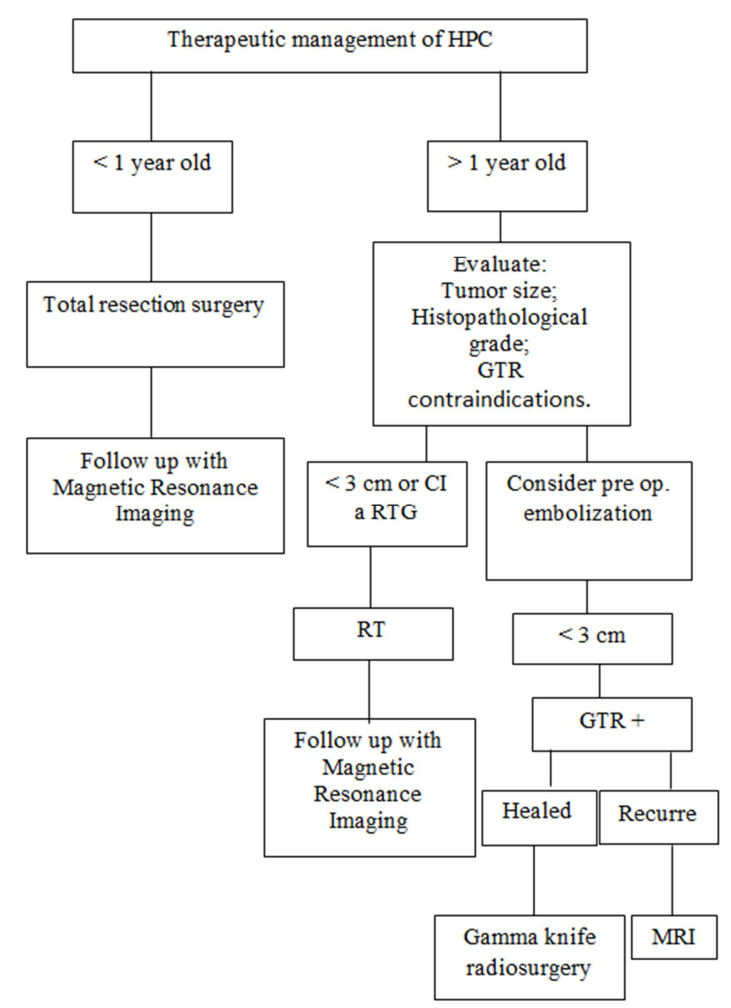

Figure 2. Treatment of hemangiopericytoma

The hemangiopericytoma is a hypervascular sarcoma of body's soft tissues. Given its rarity, it is observed a lack of studies about it. Besides that, it is noticed that the existent studies have unsatisfactory number of samples to a more clarifying conclusion.
The studies indicated a preference for performing total resection surgery associated to adjuvant radiotherapy, which would raise the survival rate and culminate in lower index of recurrences, according to the authors. However, more works should be accomplished to conclude which is the best alternative.

The approach should be individualized according to the tumor features, such as committed margins, offering the RT as therapeutic choice in cases with tumor less than $3 \mathrm{~cm}$ or with operatory contraindications, whereas eventually the GTR is not the preferred approach.

Therefore, it should be considered in the approach of hemangiopericytomas: tumor's area, size, reoccurrence ratio, patient's age and ability of satisfactory result with the chosen treatment.

In the involvement of children younger than 1 year old it is recommended the surgical treatment, and it is inconclusive the RTPO's indication. About the well-succeeded patients, a few evolve with good neuropsychomotor development after the correct treatment. Because of the young age and the possibility of recurrence, it is suggested the prolonged follow-up.

In the reported case, the suggested neurological disorder was found during the prenatal ultrasonography, resected in the first year's life and proved to be an HPC. The patient is well after one year and does follow-up with magnetic resonance imaging.

The barriers present in the review includes find studies that shows good scientific evidence given the rare character of the HPC, loss of the patients' follow-up and long follow-up period. Therefore, the conduct and the conclusions about the theme should be constantly evaluated by the time new studies are published.

\section{Conflict of Interest}

All the authors do not have any possible conflicts of interest.

\section{References}

[1] Chheda MG, Wen PY. Uncommon brain tumors. In: Eichler AF, Loeffler JS, editors. UpToDate. [Internet]. Waltman (MA): UpToDate Inc. 2019. [uptodate 2019 Feb 15; cited May 2019]. [51 screens] Available from: https://www.uptodate.com/contents/uncommon-braintumors? source=history_widget.

[2] Hayenga HN, Bishop AJ, Wardak Z, Sen C, Mickey B. Intraspinal Dissemination and Local Recurrence of an Intracranial Hemangiopericytoma. World neurosurgery. 2019; 123: 68-75.

[3] Demicco EG, Meyer C. Solitary fibrous tumor. In: Maki R, DeLaney TF, R JJ, Friedberg JS, Berman RS, Pollock RE, Shah S, Collins SA, editors. [UpToDate]. Waltham (MA). UpToDate Inc; 2019. [updated 2019 Mar 22; cited 2019 May]; [39 screens]. Available from: https://www.uptodate.com/contents/solitary-fibroustumor? source=history_widget. 
[4] Jeon SH, Park SH, Kim JW, Park CK, Paek SH, Kim IH. Efficacy of adjuvant radiotherapy in the intracranial hemangiopericytoma. Journal of Neuro-Oncology. 2018: 137 (3); 567-573.

[5] Roth J, Constantini S. 5ALA in pediatric brain tumors is not routinely beneficial. Child's Nervous System. 2017; 33 (5): 787-792.

[6] Lee, C.-K., Liu, K.-L., \& Huang, S.-K. (2019). A dedifferentiated solitary fibrous tumor of the parotid gland: a case report with Cytopathologic findings and review of the literature. Diagnostic Pathology, 14 (1).

[7] Li YQ, Chua ET, Chua KL, Chua ML. "Cor Occidere": a novel strategy of targeting the tumor core by radiosurgery in a radio- and chemo-resistant intracranial hemangiopericytoma. Chin Clin Oncol 2018; 7 (1): 10.

[8] Semerci YS., Demirel G, Vatansever B, Gundogdu S, Bolukbasi F, Oran $G$ et al. Urgent surgical management of congenital intracranial hemangiopericytoma in a preterm neonate. British Journal of Neurosurgery. 2017; $1-3$.

[9] Koc AM, Oner AY, Ucar M, Bor AO. Intracranial Hemangiopericytoma in a Child: A Case Report with Magnetic Resonance Spectroscopy Findings. Turk Neurosurg 2016; 26 (1): 173-175.

[10] Melone AG, D'Elia A, Santoro F, Salvati M, Delfini R, Cantore $\mathrm{G}$ et al. Intracranial Hemangiopericytoma-Our Experience in 30 Years: A Series of 43 Cases and Review of the Literature. World Neurosurgery. 2014; 81 (3-4): 556-562.

[11] Bridges K, Sauerwein R, Grafe MR, Tsikitis V. (Liana), Halfpenny A, Raslan AM. Seeding of Abdomen with Primary Intracranial Hemangiopericytoma by Ventriculoperitoneal Shunt: Case Report. World Neurosurgery. 2017; 10: 1048.e71048.e14.

[12] Sweid A, Noureldine MHA, Nasser S, Badra M, Saikali I. A Case Report of Meningeal Hemangiopericytoma: A 9-Year Journey from the Brain to the Spine. World neurosurgery. 2019; 122: 459-463.

[13] Wang W, Zhang GJ, Zhang LW, Li D, Wu Z, Zhang J-T. LongTerm Outcome and Prognostic Factors After Repeated Surgeries for Intracranial Hemangiopericytomas. World Neurosurgery. 2017; 107: 495-505.

[14] Campobasso D, Ferretti S, Pilato FP, Frattini A. Giant bilateral renal metastases from a meningeal hemangiopericytoma. Journal of cancer research and therapeutics. 2018; 14 (6): 1434.

[15] Towner JE, Johnson MD, Li YM. Intraventricular Hemangiopericytoma: A Case Report and Literature Review. World Neurosurgery. 2016; 89: 728.e5-728.e10.

[16] Koc AM, One AY, Ucar M, Borcek AO. Intracranial Hemangiopericytoma in a Child: A Case Report with Magnetic Resonance Spectroscopy Findings. Turkish neurosurgery. 2016; 26 (1): 173-175.

[17] Chen L, Yang Y, Yu X, Gui Q, Xu B, Zhou D. Multimodal treatment and management strategies for intracranial hemangiopericytoma. Journal of Clinical Neuroscience. 2015; 22 (4): 718-725.

[18] Ma L, Wang L, Fang X, Zhao CH, Sun L. Diagnosis and treatment of solitary fibrous tumor/hemangiopericytoma of central nervous system. Retrospective report of 17 patients and literature review. Neuroendocrinology Letters. 2017; 39 (2).

[19] Zhang G-J, Wu Z, Zhang L-W, Li D, Zhang J-T. Surgical Management and Adverse Factors for Recurrence and LongTerm Survival in Patients with Hemangiopericytoma. World Neurosurgery. 2017; 104: 95-103.

[20] Lee EJ, Kim JH, Park ES, Khang SK, Cho YH, Hong SH et al. The impact of postoperative radiation therapy on patterns of failure and survival improvement in patients with intracranial hemangiopericytoma. Journal of Neuro-Oncology. 2015; 127 (1): 181-190.

[21] Sweid A, Noureldine MHA, Nasser S, Badra M, Saikali I. A Case Report of Meningeal Hemangiopericytoma: A 9-Year Journey from the Brain to the Spine. World neurosurgery. 2019; 122: 459-463.

[22] Trifiletti DM, Mehth, GU, Grover S, Sheehan JP. Clinical management and survival of patients with central nervous system hemangiopericytoma in the National Cancer Database. Journal of Clinical Neuroscience. 2017; 44: 169-174.

[23] Choi J, Park S-H, Khang SK, Suh Y-L, Kim SP, Lee YS et al. Hemangiopericytomas in the Central Nervous System: A Multicenter Study of Korean Cases with Validation of the Usage of STAT6 Immunohistochemistry for Diagnosis of Disease. Annals of Surgical Oncology; 2016; 23 (S5): 954-96.

[24] Kamamoto D, Ohara K, Kitamura Y, Yoshida K, Kawakami Y, Sasaki H. Association between programmed cell death ligand1 expression and extracranial metastasis in intracranial solitary fibrous tumor/hemangiopericytoma. Journal of neurooncology. 2018; 139 (2): 251-259.

[25] Spina A, Boari N, Gagliardi F, Donofrio CA, Franzin A, Mortini P. The current role of Gamma Knife radiosurgery in the management of intracranial haemangiopericytoma. Acta Neurochirurgica. 2016; 158 (4): 635-642.

[26] Kim BS, Kong DS, Seol HJ, Nam DH, Lee J I. Gamma knife radiosurgery for residual or recurrent intracranial hemangiopericytomas. Journal of Clinical Neuroscience. 2017; 35: $35-41$.

[27] Shaigany K, Fang CH, Patel TD, Parque RC, Baredes S, Eloy JA. Shaigany, A population-based analysis of Head and Neck hemangiopericytoma. The Laryngoscope. 2015; 126 (3): 643650 .

[28] Jong I, Chen S, Leung YL, Cheung SK, Ho C. 11C-Acetate PET/CT in a Case of Recurrent Hemangiopericytoma. Clinical Nuclear Medicine. 2014; 39 (5): 478-479.

[29] Iampreechakul $P$, Tirakotai $W$, Lertbutsayanukul $P$, Siriwimonmas S, \& Liengudom A. Pre-operative Embolization of Intracranial and Extracranial Tumozrs: A Review of 37 Cases. Journal of the Medical Association of Thailand= Chotmaihet thangphaet. 2016; 99: S91-119.

[30] Wang X, Zhang X, Zhou Q, Zhou X, Chen L, Huang G, et al. Hemangiopericytoma Arose From the Site of Meningioma Resection. The Journal of craniofacial surgery. 2017; 28 (4): e329-e330.

[31] Tata A, Cohen-Inbar, Sheehan JP. Treatment of orbital solitary fibrous tumour with gamma knife radiosurgery and systematic review of literature. Case Reports, 2016, bcr2016217114. 
[32] Lee CK, Liu KL, Huang SK. A dedifferentiated solitary fibrous tumor of the parotid gland: a case report with Cytopathologic findings and review of the literature. Diagnostic Pathology; 2019; 14 (1). 